\title{
Non-Bayesian and Bayesian Estimation of Parameters for Burr Type III Distribution in Presence of $\boldsymbol{k}$ Outliers
}

\section{Saeed E. Hemeda and ${ }^{2}$ Ali M. Abdallah}

${ }^{1}$ Obour High Institute for Management\& Informatics, Cairo, Egypt. ${ }^{2}$ Aswan University, Quantitative Methods Department, Aswan, Egypt.

Correspondence Author: Saeed E. Hemeda, Obour High Institute for Management\& Informatics, Cairo, Egypt.

Received date: 23 February 2018, Accepted date: 1 April 2018, Online date: 15 April 2018

Copyright: (C) 2018 Saeed E. Hemeda and Ali M. Abdallah. This is an open-access article distributed under the terms of the Creative Commons Attribution License, which permits unrestricted use, distribution, and reproduction in any medium, provided the original author and source are credited.

\begin{abstract}
This paper deals with the classical (non-Bayesian) and Bayesian estimation of the Weibull distribution parameters in the presence of outliers. It estimates the unknown parameters for Burr type III distribution in the presence of $k$ outliers. Some properties of the proposed distribution are obtained such as $r$ th moment, mean, variance, mean square error, skewness and kurtosis. Moment and maximum likelihood methods are used. Furthermore, the bayesian estimation method is discussed. Estimation of the parameters for Burr type III distribution in the presence of one and two outliers are introduced using numerical studies through moment, maximum likelihood, and Bayesian methods. The paper also compares the moments, the maximum likelihood, and the Bayesian estimators.
\end{abstract}

Key words: Outliers; Burr type III distribution; Reliability; Hazard rate; Maximum likelihood; Moment; Bayesian Estimation.

\section{INTRODUCTION}

In the last few years, there has been an increasing interest in the issue of detecting possible outliers for some distributions. The presence of outliers violates the assumption that errors are normally distributed and reduces the performance of the linear regression models. Outliers may occur as a result of for many reasons including typos in data entry, scoring error, and sampling. Outliers can be described in terms of their location and impact. See Grubbs (1969). Many authors studied different probability distributions in the presence of outliers. For examples; Dixitet al. (1996) studied a set of random variables distributed according to exponentiated gamma distribution in the presence of k outliers. Hossain and Nath (1997) studied Burr XII distribution in the presence of an outlier, Dixit and Nasiri (2001) considered estimation of parameters of the exponential distribution in the presence of outliers generated from uniform distribution. Nooghabi $e t$ al. (2009) estimated the parameters of gamma distribution in the presence of outliers generated from uniform distribution. Dixit and Nooghabi (2011) studied the efficient estimation in the Pareto distribution with the presence of outliers. Deiri (2011) studied the estimation of parameters of gamma distribution in the presence of two outliers. Makhdoom (2011) discussed the estimation of the parameters of minimax distribution in the presence of outliers. Ghanizadeh et al. (2011) studied the classical estimations for the parameters of the exponentiated gamma distribution in the presence of $k$ outliers. Hassan et al. (2013) also studied the estimation of stress-strength reliability in the presence of $k$ outlier. Also, Khokan et al. (2013) discussed the weighted maximum likelihood estimation for the Weibull distribution in the presence of $k$ outlier. Gupta and Singh (2017) studied the classical and Bayesian estimation of the parameters of Weibull distribution in presence of outliers.

Burr (1942) obtained twelve distribution functions that arelisted in Burr family distributions. These distributions have yielded a variety of density shapes. Burr Types III and XII are the simplest functionally. Therefore, these two distributions are the most desirable for statistical modeling. The Burr family distributions have an important position in the field of life testing because of its uses to fit business failure data. Many well-known distributions, including the Weibull, exponential, Gompertz, normal, extreme value, logistic and uniform distributions are special cases or limited cases of Burr family distributions.

The objective of this paper is to estimate the unknown parameters forthe Burr type III distribution in the presence of $k$ outliers. The moment estimation, the maximum likelihood estimation (MLE) and the Bayesian estimation methods to estimate the shape and scale parameters of the model are used. This paper involves 4 sections. In section 1 , an introduction to Burr type III distribution is introduced. In section 2 , the joint distribution of $\left(X_{1}, X_{2}, X_{3}, \ldots, X_{n}\right)$ in the presence of $k$ outliers is generated from Burr type III distribution, and the reliability and hazard failure rate functions are presented. The estimation of the parameters using the moment, maximum likelihood and Bayesian methods are shown in Section 3. In section 5, the bias, mean square error, skewness and kurtosis are conducted using numerical studies for the moment, MLE and Bayesian methods, also conclusions and remarks based on the results of data.

\section{MATERIALS AND METHODS}

Description of the Model with $k$ Outliers:

Nasiri and Pazira (2010) assumed that $X_{1}, X_{2}, X_{3}, \ldots, X_{n}$ is a set of random variables such that $k$ outliers of them are distributed with probability density function (pdf), $g(x ; \theta, \beta)$, where

$g(x ; \theta, \beta)=\theta \beta x^{-(\beta+1)}\left(1+x^{-\beta}\right)^{-(\theta+1)} ; x>0, \theta>0, \beta>0$

Where $\theta^{\text {and } \beta}$ are the scale and shape parameters of Burr type III (BIII) distribution and the remaining $(n-k)$ random variables are distributed with (pdf), $f(x ; \propto, \lambda)$, where, 
$g(x ; \lambda, \alpha)=\lambda \alpha x^{-(\lambda+1)}\left(1+x^{-\lambda}\right)^{-(\alpha+1)} ; x>0, \lambda>0, \alpha>0$.

According to Dixit (1989), the joint distribution of $\left(X_{1}, X_{2}, \ldots, X_{n}\right)$ in thepresence of $k$ outliers could be expressed as

$f\left(x_{1}, x_{2}, \ldots, x_{n} ; \lambda, \alpha, \beta, \theta\right)=\frac{(n-k) ! k !}{n !} \prod_{i=1}^{n} f\left(x_{i}, \lambda, \alpha\right) \cdot \sum_{\underline{A}} \prod_{i=1}^{k} \frac{g\left(x_{i}, \beta, \theta\right)}{f\left(x_{i} ; \lambda, \alpha\right)}$.

By substituting from (1) and (2) in (3), the joint distribution of $\left(X_{1}, X_{2}, \ldots, X_{n}\right)$ is given by

$f\left(x_{1}, x_{2}, \ldots, x_{n} ; \lambda, \alpha, \beta, \theta\right)=\frac{(n-k) ! k !}{n !} \prod_{i=1}^{n} \lambda \alpha x_{i}^{-(\lambda+1)}\left(1+x_{i}^{-\lambda}\right)^{-(\alpha+1)}$

$$
\cdot \sum_{\underline{A}} \prod_{i=1}^{k} \frac{\beta \theta x_{A_{i}}^{-(\beta+1)}\left(1+x_{A_{i}}^{-\beta}\right)^{-(\theta+1)}}{\lambda \alpha x_{A_{i}}^{-(\lambda+1)}\left(1+x_{A_{i}}^{-\lambda}\right)^{-(\alpha+1)}},
$$

$f\left(x_{1}, x_{2}, \ldots, x_{n} ; \lambda, \alpha, \beta, \theta\right)=\frac{(n-k) ! k !}{n !} \lambda^{n-k} \alpha^{n-k} \beta^{k} \theta^{k} \cdot \prod_{i=1}^{n}\left[x_{i}^{-(\lambda+1)}\left(1+x_{i}^{-\lambda}\right)^{-(\alpha+1)}\right]$

$$
\cdot \sum_{\underline{A}}\left[\prod_{i=1}^{k} x_{A_{i}}^{\lambda-\beta} \cdot \frac{\left(1+x_{A_{i}}^{-\beta}\right)^{-(\theta+1)}}{\left(1+x_{A_{i}}^{-\lambda}\right)^{-(\alpha+1)}}\right] .
$$

Where, $\sum_{\underline{A}}=\sum_{A_{1}}^{n-k+1} \sum_{A_{2}=A_{1}+1}^{n-k+2} \ldots \sum_{A_{k}=A_{k-1}+1}^{n}$ and $k=1,2, \ldots, n$.

The marginal distribution of random variable $X$ inequation (4) is given by the following equation

$f(x ; \Phi)=\frac{n-k}{n} \lambda \alpha x^{-(\lambda+1)}\left(1+x^{-\lambda}\right)^{-(\alpha+1)}$

$$
+\frac{k}{n} \theta \beta x^{-(\beta+1)}\left(1+x^{-\beta}\right)^{-(\theta+1)} ; x>0, \lambda, \alpha, \theta, \beta>0 .
$$

Where, $\Phi$ is a parameters vector and $\Phi=(\lambda, \alpha, \beta, \theta)$ (Dixit et al.(1996) and Nasiri and Pazira (2010)).The corresponding cdf of pdf (5) is $F(x ; \Phi)=\frac{n-k}{n}\left(1+x^{-\lambda}\right)^{-\alpha}+\frac{k}{n}\left(1+x^{-\beta}\right)^{-\theta} \cdot{ }_{(6)}$

The Reliability and hazard rate functions are, respectively

$R(x ; \Phi)=1-\frac{n-k}{n}\left(1+x^{-\lambda}\right)^{-\alpha}-\frac{k}{n}\left(1+x^{-\beta}\right)^{-\theta}$,

$H(x ; \Phi)=\frac{\frac{n-k}{n} \lambda \alpha x^{-(\lambda+1)}\left(1+x^{-\lambda}\right)^{-(\alpha+1)}+\frac{k}{n} \theta \beta x^{-(\beta+1)}\left(1+x^{-\beta}\right)^{-(\theta+1)}}{1-\frac{n-k}{n}\left(1+x^{-\lambda}\right)^{-\alpha}-\frac{k}{n}\left(1+x^{-\beta}\right)^{-\theta}}$.

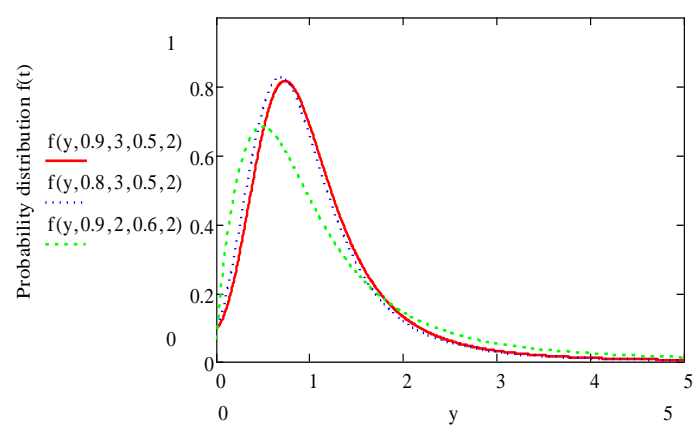

time

Burr type III pdf

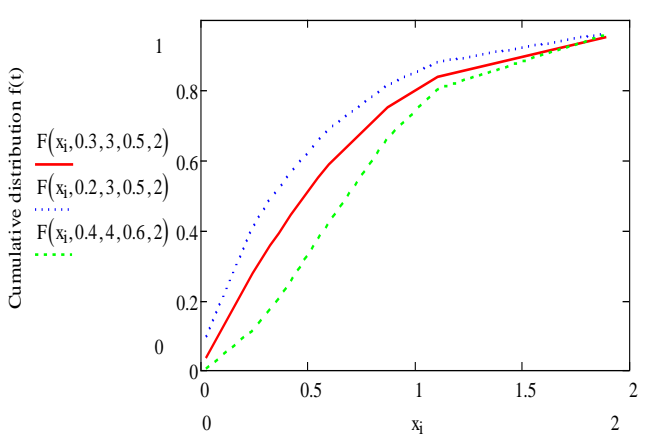

time

Burr type III cdf 


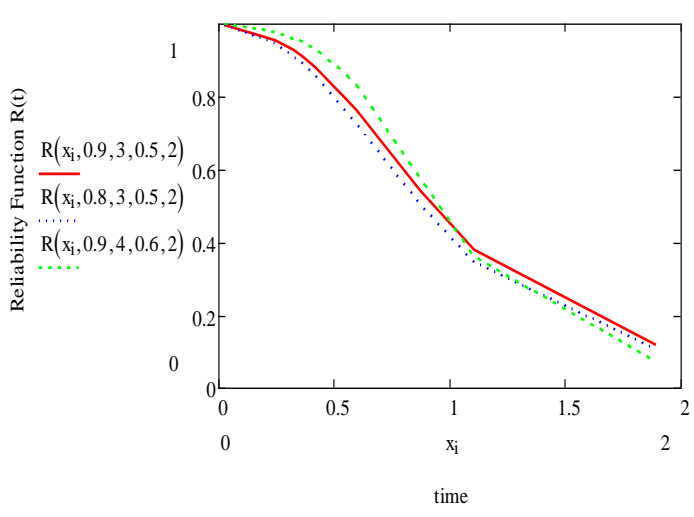

Burr type III Reliability

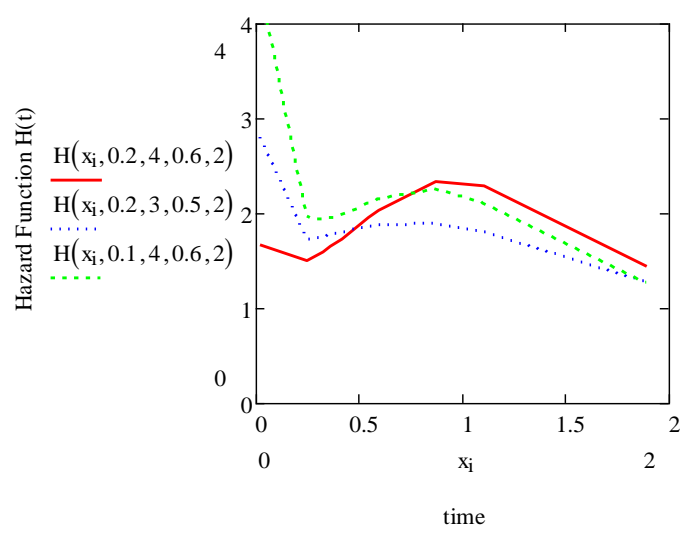

Burr type III Hazard

Fig. 1: The pdf, cdf, reliability and hazard rate functions of BIII for selected values of the parameters.

\section{Parameters Estimation:}

In this section, the parameters of Burr type III distribution in the presence of $k$ outliers are estimated using the non-bayesian methods (moment, maximum likelihood) and the Bayesian method.

i. Moment Method:

The $\mathrm{r}^{\text {th }}$ moment of BIII distribution in the presence of $k$ outliers can be obtained using the pdf (5) as follows

$$
\begin{aligned}
& E\left(X^{r}\right)=\mu_{r}^{\prime}=\int_{0}^{\infty} x^{r} f(x ; \Phi) d x, \\
& \mu^{\prime}=\frac{n-k^{\infty}}{n} \int_{0}^{\infty} x^{r} \lambda \alpha x^{-(\lambda+1)}\left(1+x^{-\lambda}\right)^{-(\alpha+1)} d x+\frac{k}{n} \int_{0}^{\infty} \theta \beta x^{-(\beta+1)}\left(1+x^{-\beta}\right)^{-(\theta+1)} d x, \\
& \mu_{r}^{\prime}=\frac{n-k}{n}\left[\alpha \cdot B\left(1-\frac{r}{\lambda}, \alpha+\frac{r}{\lambda}\right)\right]+\frac{n-k}{n}\left[\theta \cdot B\left(1-\frac{r}{\beta}, \theta+\frac{r}{\beta}\right)\right], \\
& \mu_{r}^{\prime}=\frac{n-k}{n}\left[\frac{\Gamma\left(1-\frac{r}{\lambda}\right) \Gamma\left(\alpha+\frac{r}{\lambda}\right)}{\Gamma(\alpha)}\right]+\frac{k}{n}\left[\frac{\Gamma\left(1-\frac{r}{\beta}\right) \Gamma\left(\theta+\frac{r}{\beta}\right)}{\Gamma(\theta)}\right] ; r<\lambda, r<\beta .
\end{aligned}
$$

When $r=1,2$ we can obtain the mean and the variance of BIII distribution in the presence of $k$ outliersas follows;

The $1^{\text {st }}$ moment (mean) is

$\mu_{1}^{\prime}=E(X)=\frac{n-k}{n}\left[\frac{\Gamma\left(1-\frac{1}{\lambda}\right) \Gamma\left(\alpha+\frac{1}{\lambda}\right)}{\Gamma(\alpha)}\right]+\frac{k}{n}\left[\frac{\Gamma\left(1-\frac{1}{\beta}\right) \Gamma\left(\theta+\frac{1}{\beta}\right)}{\Gamma(\theta)}\right] ; \lambda>1, \beta>1$.

The $2^{\text {nd }}$ moment is

$$
\mu_{2}^{\prime}=E\left(X^{2}\right)=\frac{n-k}{n}\left[\frac{\Gamma\left(1-\frac{2}{\lambda}\right) \Gamma\left(\alpha+\frac{2}{\lambda}\right)}{\Gamma(\alpha)}\right]+\frac{k}{n}\left[\frac{\Gamma\left(1-\frac{2}{\beta}\right) \Gamma\left(\theta+\frac{2}{\beta}\right)}{\Gamma(\theta)}\right] .
$$

The variance is

$\operatorname{Var}(X)=\frac{n-k}{n}\left[\frac{\Gamma\left(1-\frac{2}{\lambda}\right) \Gamma\left(\alpha+\frac{2}{\lambda}\right)}{\Gamma(\alpha)}\right]+\frac{k}{n}\left[\frac{\Gamma\left(1-\frac{2}{\beta}\right) \Gamma\left(\theta+\frac{2}{\beta}\right)}{\Gamma(\theta)}\right]-\left[\frac{n-k}{n}\left[\frac{\Gamma\left(1-\frac{1}{\lambda}\right) \Gamma\left(\alpha+\frac{1}{\lambda}\right)}{\Gamma(\alpha)}\right]+\frac{k}{n}\left[\frac{\Gamma\left(1-\frac{1}{\beta}\right) \Gamma\left(\theta+\frac{1}{\beta}\right)}{\Gamma(\theta)}\right]\right]^{2}$

\section{ii. Maximum Likelihood Method:}

The log likelihood function of sample nobservations, $\left(X_{1}, X_{2}, \ldots, X_{n}\right)$ for BIII distribution in the presence of $k$ outliers is given by

$$
L(\lambda, \alpha, \beta, \theta)=\ln f\left(x_{1}, x_{2}, \ldots, x_{n} ; \lambda, \alpha, \beta, \theta\right)
$$

Using equation (4), the likelihood function is

$$
\begin{array}{r}
L(\lambda, \alpha, \beta, \theta)=(n-k) \ln \lambda+(n-k) \ln \alpha+k \ln \theta+k \ln \beta-\ln v-(\lambda+1) \sum_{i=1}^{n} \ln x_{i} \\
-(\alpha+1) \sum_{i=1}^{n} \ln \left(1+x_{i}^{-\lambda}\right)+\ln \left[\sum_{A} \prod_{i=1}^{k} \frac{x_{A_{i}}^{\lambda-\beta}\left(1+x_{A_{i}}^{-\beta}\right)^{-(\theta+1)}}{\left(1+x_{A_{i}}^{-\lambda}\right)^{-(\alpha+1)}}\right] .
\end{array}
$$


Where, $v=\frac{n !}{k !(n-k) !}$ and $\sum_{\underline{A}}=\sum_{A_{1}}^{n-k+1} \sum_{A_{2}=A_{1}+1}^{n-k+2} \ldots \sum_{A_{k}=A_{k-1}+1}^{n}$

By taking the derivatives $\left(\frac{\partial L}{\partial \lambda}, \frac{\partial L}{\partial \alpha}, \frac{\partial L}{\partial \beta}, \frac{\partial L}{\partial \theta}\right)$ and equating to 0 , we obtain the normal equations as follows

$\frac{\partial L}{\partial \lambda}=\frac{(n-k)}{\lambda}-\sum_{i=1}^{n} \ln \left(x_{i}\right)+(\alpha+1) \sum_{i=1}^{n} \frac{x_{i}^{-\lambda} \ln \left(x_{i}\right)}{\left(1+x_{i}^{-\lambda}\right)}+\frac{\partial}{\partial \lambda} \ln \left[\sum_{A} \prod_{i=1}^{k} \frac{x_{A_{i}}^{\lambda-\beta}\left(1+x_{A_{i}}^{-\beta}\right)^{-(\theta+1)}}{\left(1+x_{A_{i}}^{-\lambda}\right)^{-(\alpha+1)}}\right]$.

$\frac{\partial L}{\partial \alpha}=\frac{(n-k)}{\alpha}-\sum_{i=1}^{n} \ln \left(1+x_{i}^{-\lambda}\right)+\frac{\partial}{\partial \alpha} \ln \left[\sum_{\underline{A}} \prod_{i=1}^{k} \frac{x_{A_{i}}{ }^{\lambda-\beta}\left(1+x_{A_{i}}{ }^{-\beta}\right)^{-(\theta+1)}}{\left(1+x_{A_{i}}{ }^{-\lambda}\right)^{-(\alpha+1)}}\right]$

$\frac{\partial L}{\partial \beta}=\frac{k}{\beta}+\frac{\partial}{\partial \beta} \ln \left[\sum_{A} \prod_{i=1}^{k} \frac{x_{A_{i}}^{\lambda-\beta}\left(1+x_{A_{i}}^{-\beta}\right)^{-(\theta+1)}}{\left(1+x_{A_{i}}^{-\lambda}\right)^{-(\alpha+1)}}\right]$.

$\frac{\partial L}{\partial \theta}=\frac{k}{\theta}+\frac{\partial}{\partial \theta} \ln \left[\sum_{\underline{A}} \prod_{i=1}^{k} \frac{x_{A_{i}}^{\lambda-\beta}\left(1+x_{A_{i}}^{-\beta}\right)^{-(\theta+1)}}{\left(1+x_{A_{i}}^{-\lambda}\right)^{-(\alpha+1)}}\right]$

Since it is difficult to solve the nonlinear equations (14), (15), (16) and (17) to obtain $\lambda, \alpha, \beta$ and $\theta$ in closed form, therefore the numerical method is used to solve the nonlinear equations simultaneously to obtained $\hat{\alpha}, \hat{\lambda}, \hat{\beta}$ and $\hat{\theta}$, see Ghanizadeh et al.(2011).

iii. Bayesian Method:

The Problem of bayesian estimation method for Burr type III distribution discussed by Abd-Elfattah and Alharbey (2012) based on trimmed samples. In this section, the Bayesian estimators and posterior variance of the shape parameters for Burr type III distribution in the presence of $k$ outliers are obtained in the case of non-informative prior. The proposed estimators are obtained under symmetric and asymmetric loss functions. The posterior mean and the posterior variance of the estimators for the shape parameters are obtained numerically. Abd-Elfattah and Alharbey (2012) discussed the bayesian estimation for Burr type III distribution based on trimmed samples. Assuming that the parameters $\lambda$ and $\beta$ are known and the parameters $\alpha$ and $\theta$ have independent prior distribution. The noninformative prior (NIP) for $\alpha$ and $\theta$ are given by

$$
\begin{array}{ll}
u_{1} \propto \alpha^{-1} & , \alpha>0 \\
u_{2} \propto \theta^{-1} & , \theta>0
\end{array}
$$

Consequently, the joint (NIP) will be

$$
u(\alpha, \theta) \propto(\alpha \theta)^{-1}, \quad \alpha, \theta>0
$$

The joint posterior density functions of $\alpha, \theta$ will be

$$
g(\alpha, \theta \mid x)=\frac{u(\alpha, \theta) L(x \mid \alpha, \theta)}{\xi}, \alpha>0, \theta>0
$$

$g(\alpha, \theta \mid x)=\frac{(\alpha \theta)^{-1} \lambda^{n-k} \alpha^{n-k} \beta^{k} \theta^{k} \prod_{i=1}^{n}\left[x_{i}^{-(\lambda+1)}\left(1+x_{i}^{-\lambda}\right)^{-(\alpha+1)}\right] \cdot \sum_{\underline{A}}\left[\prod_{i=1}^{k} \frac{x_{A_{i}}^{\lambda-\beta}\left(1+x_{A_{i}}^{-\beta}\right)^{-(\theta+1)}}{\left(1+x_{A_{i}}^{-\lambda}\right)^{-(\alpha+1)}}\right]}{\xi}$

$$
\text { ; } \alpha>0, \theta>0 \text {. }
$$

Where $\xi$ is the normalized constant defined by

$$
\xi=\int_{0}^{\infty} \int_{0}^{\infty}(\alpha \theta)^{-1} \lambda^{n-k} \alpha^{n-k} \beta^{k} \theta^{k} \prod_{i=1}^{n}\left[x_{i}^{-(\lambda+1)}\left(1+x_{i}^{-\lambda}\right)^{-(\alpha+1)}\right] \sum_{\underline{A}}\left[\prod_{i=1}^{k} \frac{x_{A_{i}}^{\lambda-\beta}\left(1+x_{A_{i}}{ }^{-\beta}\right)^{-(\theta+1)}}{\left(1+x_{A_{i}}{ }^{-\lambda}\right)^{-(\alpha+1)}}\right] d \alpha d \theta .
$$

The marginal posterior of one parameter is obtained by integrating the joint posterior distribution with respect to the other parameter, thereforethe marginal posterior probability density function of $\alpha$ is defined by

$$
g_{1}(\alpha \mid \theta, x)=\frac{\lambda^{n-k} \alpha^{n-k-1} \beta^{k} \prod_{i=1}^{n}\left[x_{i}^{-(\lambda+1)}\left(1+x_{i}^{-\lambda}\right)^{-(\alpha+1)}\right] \cdot \int_{0}^{\infty} \theta^{k-1} \cdot \sum_{\underline{A}}\left[\prod_{i=1}^{k} \frac{x_{A_{i}}^{\lambda-\beta}\left(1+x_{A_{i}}^{-\beta}\right)^{-(\theta+1)}}{\left(1+x_{A_{i}}^{-\lambda}\right)^{-(\alpha+1)}}\right] d \theta}{\xi} .
$$

Similarly, the marginal posterior of $\theta$ is defined by

$$
g_{2}(\theta \mid \alpha, x)=\frac{\lambda^{n-k} \theta^{k-1} \beta^{k} \int_{0}^{\infty} \alpha^{n-k-1} \prod_{i=1}^{n}\left[x_{i}^{-(\lambda+1)}\left(1+x_{i}^{-\lambda}\right)^{-(\alpha+1)}\right] \cdot \sum_{A}\left[\prod_{i=1}^{k} \frac{x_{A_{i}}^{\lambda-\beta}\left(1+x_{A_{i}}{ }^{-\beta}\right)^{-(\theta+1)}}{\left(1+x_{A_{i}}{ }^{-\lambda}\right)^{-(\alpha+1)}}\right] d \alpha}{\xi} .
$$

Since, it is known that under the squared error loss function, the Bayes estimator of the parameter will be its posterior expectation. The posterior mean and posterior variance of the shape parameters $\alpha$ and $\theta$ are expressed as follows 


$$
\begin{aligned}
& \tilde{\alpha}=E(\alpha \mid \theta, x)=\int_{0}^{\infty} \alpha g_{1}(\alpha \mid \theta, x) d \alpha \\
& \tilde{\alpha}=\lambda^{n-k} \beta^{k} \int_{0}^{\infty} \int_{0}^{\infty} \alpha^{n-k} \theta^{k-1} \prod_{i=1}^{n}\left[x_{i}^{-(\lambda+1)}\left(1+x_{i}^{-\lambda}\right)^{-(\alpha+1)}\right] \cdot \sum_{\underline{A}}\left[\prod_{i=1}^{k} \frac{x_{A_{i}}^{\lambda-\beta}\left(1+x_{A_{i}}^{-\beta}\right)^{-(\theta+1)}}{\left(1+x_{A_{i}}^{-\lambda}\right)^{-(\alpha+1)}}\right] d \alpha d \theta . \quad \operatorname{var}(\alpha \mid \theta, x)=E(\tilde{\alpha}-\alpha)^{2}, \\
& \operatorname{var}(\tilde{\alpha} \mid \theta, x)=\lambda^{n-k} \beta^{k} \int_{0}^{\infty} \int_{0}^{\infty}(\alpha-\tilde{\alpha})^{2} \alpha^{n-k-1} \theta^{k-1} \prod_{i=1}^{n}\left[x_{i}^{-(\lambda+1)}\left(1+x_{i}^{-\lambda}\right)^{-(\alpha+1)}\right] \\
& \sum_{\underline{A}}\left[\prod_{i=1}^{k} \frac{x_{A_{i}}^{\lambda-\beta}\left(1+x_{A_{i}}^{-\beta}\right)^{-(\theta+1)}}{\left(1+x_{A_{i}}{ }^{-\lambda}\right)^{-(\alpha+1)}}\right] d \alpha d \theta \\
& \tilde{\theta}=E(\theta \mid \alpha, x)=\int_{0}^{\infty} \theta g_{2}(\theta \mid \alpha, x) d \theta \\
& \tilde{\theta}=\lambda^{n-k} \beta^{k} \int_{0}^{\infty} \int_{0}^{n-k-1} \theta^{k} \prod_{i=1}^{n}\left[x_{i}^{-(\lambda+1)}\left(1+x_{i}^{-\lambda}\right)^{-(\alpha+1)}\right] \cdot \sum_{\underline{A}}\left[\prod_{i=1}^{k} \frac{x_{A_{i}}^{\lambda-\beta}\left(1+x_{A_{i}}^{-\beta}\right)^{-(\theta+1)}}{\left(1+x_{A_{i}}^{-\lambda}\right)^{-(\alpha+1)}}\right] d \alpha d \theta . \\
& \operatorname{var}(\theta \mid \alpha, x)=E(\tilde{\theta}-\theta)^{2}, \\
& \operatorname{var}(\tilde{\theta} \mid \alpha, x)=\lambda^{n-k} \beta^{k} \int_{0}^{\infty}(\theta-\tilde{\theta})^{2} \alpha^{n-k-1} \theta^{k-1} \prod_{i=1}^{n}\left[x_{i}^{-(\lambda+1)}\left(1+x_{i}^{-\lambda}\right)^{-(\alpha+1)}\right] \\
& \sum_{\underline{A}}\left[\prod_{i=1}^{k} \frac{x_{A_{i}}^{\lambda-\beta}\left(1+x_{A_{i}}^{-\beta}\right)^{-(\theta+1)}}{\left(1+x_{A_{i}}^{-\lambda}\right)^{-(\alpha+1)}}\right] d \alpha d \theta
\end{aligned}
$$

Equations $(22,23,24$ and 25$)$ are very difficult to be exactly solved, therefore iterative procedure is adopted to solve these equations numerically using the Mathematica statistical package. The posterior mean and the posterior variance of the shape parameters $(\alpha, \theta)$ are obtained numerically.

Numerical Study:

In this section, estimation of the parameters for Burr type III distribution in the presence of one and two outliers reconducted using moment, ML and Bayesian estimation methods.

\section{i. Moment numerical illustration:}

In this subsection, the moment estimation method for the parameters of the Burr type III distribution in the presence of $k$ outliers is discussed. Random numbers are generated for Burr type III (with and without outliers) performing sampling experiments using Mathcad (14). The estimators for $\lambda, \alpha, \beta$ and $\theta$ are obtained. Values of biases and MSEs of these estimates are calculated based on 100 replications using a sample of $\operatorname{size}(n=25,50,75,100)$ where $k=1,2$ for $\alpha=$ $0.2, \lambda=2.8, \beta=2.9, \theta=0.3$. Results for selected values of the parameters are presented in tables (1) and (2).

\section{ii. Maximum Likelihood numerical illustration:}

To check bias and mean square error of the moment method, random numbers from the Burr type III distribution are generated to perform sampling experiments using Mathcad (14). We obtained the estimators $\hat{\lambda}, \hat{\alpha}, \hat{\beta}$ for $\lambda, \alpha, \beta$, respectively. Values of biases and $\mathrm{MSE}_{\mathrm{s}}$ for these estimates are reported based on 100 replications with sample size $(n=25,50,75,100)$ where $k=1,2$ for $\alpha=0.2, \lambda=2.8, \beta=2.9, \theta=0.3$. Results for selected values of the parameters are presented in tables (3) and (4).

\section{iii. Bayesian numerical illustration:}

Properties of the derived estimators could be studied theoretically or analytically. usually it is very difficult to study the properties of the estimators' theoretically because of the complicated formula of the estimators. Therefore, a numerical study will be set up, treating separately the sampling distribution of the estimators. Steps for the numerical studyare introduced in the following:

Step (1):

Generate a random sample of size 10, 20,40,60,80 and 100 from Burr type III distribution. The generation of Burr type III distribution is very simple if $U$ has a uniform $(0,1)$ random number, then $X_{1}=\left(U^{-\frac{1}{\alpha}}-1\right)^{-\frac{1}{\lambda}}$ and $X_{2}=\left(U^{-\frac{1}{\theta}}-1\right)^{-\frac{1}{\beta}}$ follow Burr type III distributions, and then $X=\frac{n-k}{n} X_{1}+\frac{k}{n} X_{2}$ follows Burr type III distribution. The true parameters selected values are $(\alpha=1, \theta=2.5)$.

Step (2):

Solve the nonlinear equations to obtain the estimators $(\tilde{\alpha}, \tilde{\theta})$ using equations $((4.33),(4.34))$ and the posterior variances $(\operatorname{var}(\tilde{\alpha}), \operatorname{var}(\tilde{\theta}))$ of the shape and scale parameters $(\alpha, \theta)$ using (4.35) and (4.36).

Step (3):

The posterior mean, bias, MSE, skewness and kurtosis of the estimators of parameters $(\alpha, \theta)$ for sample size $(n=10,20,30,40$ and 50$)$ in the case of one outlier $(k=1)$ are introduced in table (5). For the case of two outliers $(k=2)$, table (6) summarizes the results. 
Citation: Saeed E. Hemeda and Ali M. Abdallah, 2018. Non-Bayesian and Bayesian Estimation of Parameters for Burr Type III Distribution in Presence of K Outliers. Australian Journal of Basic and Applied Sciences., 12(4): 5-13.

Table 1: Parameters moment estimation for Burr type III distribution in presence of one outlier with initial values; $k=1, \alpha=0.4, \lambda=1.8, \theta=0.3, \beta=1.9$.

\begin{tabular}{|c|c|c|c|c|c|c|c|}
\hline$n$ & Parameter & Mean & Bias & MSE & SE & Skewness & Kurtosis \\
\hline \multirow{4}{*}{25} & $\alpha$ & 0.40413 & -0.00413 & 0.00270 & 0.00207 & 0.568 & 2.069 \\
\hline & $\lambda$ & 2.01694 & -0.21694 & 0.21118 & 0.0162 & 1.268 & 3.483 \\
\hline & $\theta$ & 0.24908 & 0.05092 & 0.00803 & 0.00295 & -1.017 & 2.509 \\
\hline & $\beta$ & 1.03774 & 0.86226 & 1.09868 & 0.02384 & 0.19 & 1.352 \\
\hline \multirow{4}{*}{50} & $\alpha$ & 0.41279 & -0.01279 & 0.00080 & 0.0005 & -0.386 & 2.114 \\
\hline & $\lambda$ & 1.8631 & -0.0631 & 0.02499 & 0.0029 & 0.603 & 2.061 \\
\hline & $\theta$ & 0.2908 & 0.0092 & 0.01118 & 0.00211 & 0.771 & 3.623 \\
\hline & $\beta$ & 0.9327 & 0.9673 & 1.26121 & 0.01141 & 0.615 & 1.804 \\
\hline \multirow{4}{*}{75} & $\alpha$ & 0.41466 & -0.01466 & 0.00070 & 0.00029 & -1.003 & 3.059 \\
\hline & $\lambda$ & 1.7964 & 0.0036 & 0.00933 & 0.00129 & $\mid-0.081$ & 2.293 \\
\hline & $\theta$ & 0.28225 & \begin{tabular}{|l|}
0.01775 \\
\end{tabular} & 0.00190 & 0.00053 & -2.12 & 6.051 \\
\hline & $\beta$ & 1.21893 & 0.68107 & 0.80592 & 0.0078 & 0.164 & 1.065 \\
\hline \multirow{4}{*}{100} & $\alpha$ & 0.41161 & -0.01161 & 0.00056 & 0.00021 & 0.505 & 2.03 \\
\hline & $\lambda$ & 1.8633 & -0.0633 & 0.01292 & 0.00094 & -0.047 & 1.569 \\
\hline & $\theta$ & 0.28436 & 0.01564 & 0.00219 & 0.00044 & -2.242 & 6.465 \\
\hline & $\beta$ & 1.24799 & 0.65201 & 0.90689 & 0.00694 & -0.151 & 0.975 \\
\hline
\end{tabular}

Table 2: Parameters moment estimation for Burr type III distribution in presence of twooutliers with initial values; $k=2, \alpha=0.4, \lambda=1.8, \theta=0.3, \beta=1.9$.

\begin{tabular}{|c|c|c|c|c|c|c|c|}
\hline$n$ & Parameter & Mean & Bias & MSE & SE & Skewness & Kurtosis \\
\hline \multirow{4}{*}{25} & $\alpha$ & 0.05404 & 0.14596 & 0.02314 & 0.00171 & 1.9690 & \begin{tabular}{|l|l|}
5.719 \\
\end{tabular} \\
\hline & $\lambda$ & 1.3697 & 1.4303 & 2.04632 & 0.00631 & 0.2069 & 1.633 \\
\hline & $\theta$ & 0.09152 & 0.20848 & 0.04923 & 0.00304 & 2.0661 & 6.053 \\
\hline & $\beta$ & 1.50803 & 1.39197 & 1.96406 & 0.00651 & 2.0420 & 5.912 \\
\hline \multirow{4}{*}{50} & $\alpha$ & 0.05921 & 0.14079 & 0.02078 & 0.00062 & 2.2020 & 6.371 \\
\hline & $\lambda$ & 1.36235 & 1.43765 & 2.06714 & 0.00431 & 0.3989 & 2.635 \\
\hline & $\theta$ & 0.08135 & 0.21865 & 0.04822 & 0.00041 & 2.2745 & 6.563 \\
\hline & $\beta$ & 1.4846 & 1.4154 & 2.00618 & 0.00106 & -0.7580 & 3.912 \\
\hline \multirow{4}{*}{75} & $\alpha$ & 0.20176 & |-0.0017 & 0.03462 & 0.00248 & 1.2670 & 3.566 \\
\hline & $\lambda$ & 1.35892 & 1.44108 & 2.07732 & 0.00423 & 0.9793 & 2.75 \\
\hline & $\theta$ & 0.22994 & 0.07006 & 0.05021 & 0.00284 & 0.3448 & 0.963 \\
\hline & $\beta$ & 1.69355 & 1.20645 & 1.52541 & 0.00352 & 0.1270 & 1.087 \\
\hline \multirow{2}{*}{100} & $\alpha$ & 0.14645 & 0.05355 & 0.01512 & 0.00111 & 0.9860 & 2.795 \\
\hline & $\lambda$ & 1.33933 & 1.46067 & 2.13604 & 0.00310 & -1.9930 & 5.734 \\
\hline
\end{tabular}

Table 3: MLE of parameters estimation for Burr type III in presence of one outlier with initial values: $k=1, \alpha=0.5, \lambda=2.8, \theta=0.4, \beta=2.9$.

\begin{tabular}{|c|c|c|c|c|c|c|c|}
\hline$n$ & Parameter & Mean & Bias & MSE & SE & Skewness & Kurtosis \\
\hline \multirow{4}{*}{25} & $\alpha$ & 0.47904 & -0.07904 & 0.01429 & 0.00359 & 0.198 & 1.393 \\
\hline & $\lambda$ & 1.9321 & -0.1321 & 0.12704 & 0.01324 & -0.431 & 1.881 \\
\hline & $\theta$ & 0.06486 & 0.23514 & 0.05529 & 0.00006 & -0.191 & 1.489 \\
\hline & $\beta$ & 2.87876 & -0.97876 & 1.62621 & 0.0327 & -0.074 & 1.372 \\
\hline \multirow{4}{*}{50} & $\alpha$ & 0.45982 & -0.05982 & 0.01579 & 0.00221 & 0.232 & 2.946 \\
\hline & $\lambda$ & 1.89967 & -0.09967 & 0.26268 & 0.01005 & 1.497 & 4.659 \\
\hline & $\theta$ & 0.06421 & 0.23579 & 0.05560 & 0.00002 & 0.123 & 1.464 \\
\hline & $\beta$ & 2.14421 & -0.24421 & 0.50851 & 0.0134 & -0.012 & 1.29 \\
\hline \multirow{3}{*}{75} & $\alpha$ & 0.40517 & -0.00517 & 0.00903 & 0.00127 & "-0.126 & 1.792 \\
\hline & $\lambda$ & 2.12294 & -0.32294 & 0.55421 & 0.00894 & 0.975 & 2.477 \\
\hline & $\theta$ & 0.06444 & 0.23556 & 0.05549 & 0.00001 & -1.561 & 4.926 \\
\hline
\end{tabular}


Citation: Saeed E. Hemeda and Ali M. Abdallah, 2018. Non-Bayesian and Bayesian Estimation of Parameters for Burr Type III Distribution in Presence of K Outliers. Australian Journal of Basic and Applied Sciences., 12(4): 5-13.

\begin{tabular}{|c|c|c|c|c|c|c|c|}
\hline \hline & $\beta$ & 2.04272 & -0.14272 & 0.25710 & 0.00649 & -0.926 & 3.175 \\
\hline \hline \multirow{3}{*}{100} & $\alpha$ & 0.39413 & 0.00587 & 0.00499 & 0.0007 & -0.021 & 1.228 \\
\cline { 2 - 8 } & $\lambda$ & 1.93065 & -0.01306 & 0.08094 & 0.00253 & 1.21 & 3.414 \\
\cline { 2 - 8 } & $\theta$ & 0.06505 & 0.23495 & 0.05520 & 0.00001 & -0.00531 & 1.3921 \\
\cline { 2 - 8 } & $\beta$ & 1.91391 & -0.01391 & 0.10584 & 0.00325 & -0.1066 & 2.2225 \\
\hline \hline
\end{tabular}

Table 4: MLE of parameters estimation for Burr type III in presence of two outliers with initial values; $k=1, \alpha=0.5, \lambda=2.8, \theta=0.4, \beta=2.9$.

\begin{tabular}{|c|c|c|c|c|c|c|c|}
\hline$n$ & Parameter & Mean & Bias & MSE & SE & Skewness & Kurtosis \\
\hline \multirow{4}{*}{25} & $\alpha$ & 0.50681 & -0.00681 & 0.00673 & 0.00327 & 1.075 & 3.56 \\
\hline & $\lambda$ & 3.06868 & -0.26868 & 0.18243 & 0.0146 & 0.642 & 2.53 \\
\hline & $\theta$ & 0.42988 & -0.02988 & 0.00679 & 0.00307 & 0.025 & 2.674 \\
\hline & $\beta$ & 3.36845 & -0.46845 & 1.12869 & 0.03814 & 1.309 & 3.724 \\
\hline \multirow{4}{*}{50} & $\alpha$ & 0.51052 & -0.01052 & 0.00062 & 0.00045 & "-0.352 & 1.522 \\
\hline & $\lambda$ & 2.82554 & -0.02554 & 0.04014 & 0.00493 & 0.376 & 1.657 \\
\hline & $\theta$ & 0.39113 & 0.00887 & 0.00231 & 0.00094 & -1.988 & 5.729 \\
\hline & $\beta$ & 3.13522 & -0.23522 & 0.43260 & 0.01228 & 2.11 & 6.072 \\
\hline \multirow{4}{*}{75} & $\alpha$ & 0.51363 & -0.01363 & 0.00203 & 0.00057 & 0.989 & 2.988 \\
\hline & $\lambda$ & 2.79947 & 0.00053 & 0.04765 & 0.00968 & -0.137 & 1.531 \\
\hline & $\theta$ & 0.38084 & 0.01916 & 0.00281 & 0.00066 & -0.961 & 2.29 \\
\hline & $\beta$ & 3.20231 & -0.30231 & 0.62916 & 0.00978 & 1.912 & 5.427 \\
\hline \multirow{4}{*}{100} & $\alpha$ & 0.49219 & 0.00781 & 0.00179 & 0.00042 & -0.99 & 3.369 \\
\hline & $\lambda$ & 2.78026 & 0.01974 & 0.02808 & 0.00166 & 0.209 & 1.755 \\
\hline & $\theta$ & 0.40123 & -0.00123 & 0.00022 & 0.00015 & -0.367 & 3.364 \\
\hline & $\beta$ & 2.94518 & -0.04518 & 0.22858 & 0.00476 & 1.001 & 4.192 \\
\hline
\end{tabular}

Table 5: Bayesian parameters estimation for Burr type III in presence of one outlier with initial values; $k=1, \alpha=1, \lambda=2.8, \theta=2.5, \beta=2.9$.

\begin{tabular}{|c|c|c|c|c|c|c|}
\hline$n$ & Parameter & Mean & Bias & MSE & Skewness & Kurtosis \\
\hline \multirow[t]{2}{*}{10} & $\alpha$ & 0.37591 & 0.17591 & 0.040143 & 1.58978 & 0.0318 \\
\hline & $\theta$ & 3.47498 & -0.02502 & 6.833690 & 1.06886 & 1.74068 \\
\hline \multirow[t]{2}{*}{20} & $\alpha$ & "1.11168 & 0.11168 & 0.090571 & 0.978662 & 2.9872 \\
\hline & $\theta$ & 5.842912 & -5.34293 & 7.051123 & 0.67173 & 0.67901 \\
\hline \multirow[t]{2}{*}{30} & $\alpha$ & 0.30234 & 0.07904 & 0.031208 & 1.00123 & 1.5673 \\
\hline & $\theta$ & 2.98799 & -0.13217 & 1.000213 & 0.00547 & 4.6321 \\
\hline \multirow[t]{2}{*}{40} & $\alpha$ & 1.42101 & 0.23514 & 0.010571 & 0.012006 & 2.500632 \\
\hline & $\theta$ & 3.87618 & -0.97876 & 3.000426 & 1.10231 & 3.9813 \\
\hline \multirow[t]{2}{*}{50} & $\alpha$ & 0.24045 & -0.05981 & 0.015792 & 0.00221 & 0.232 \\
\hline & $\theta$ & 3.23466 & 0.00238 & 1.012363 & 0.00421 & 1.56409 \\
\hline \multirow[t]{2}{*}{60} & $\alpha$ & 1.19967 & 0.09967 & 0.262682 & 0.01005 & 1.497 \\
\hline & $\theta$ & 2.76421 & 0.23579 & 0.055600 & 0.00002 & 0.123 \\
\hline \multirow[t]{2}{*}{70} & $\alpha$ & 1.10760 & -0.07904 & 0.003241 & 0.00670 & 1.0643 \\
\hline & $\theta$ & 2.43005 & 0.13213 & 0.010823 & 1.09140 & 1.00461 \\
\hline \multirow[t]{2}{*}{80} & $\alpha$ & 1.00003 & 0.23514 & 0.010049 & 0.08125 & 0.9875 \\
\hline & $\theta$ & 2.48000 & -0.97876 & 0.007123 & 0.31002 & 2.08701 \\
\hline \multirow[t]{2}{*}{90} & $\alpha$ & 1.38991 & -0.05982 & 1.067518 & 0.09173 & 1.76431 \\
\hline & $\theta$ & 2.62221 & -0.09967 & 0.005002 & 0.00764 & 2.16408 \\
\hline \multirow[t]{2}{*}{100} & $\alpha$ & 1.20345 & 0.23579 & 0.000121 & 1.00618 & 1.22814 \\
\hline & $\theta$ & 2.50804 & -0.24421 & 0.002106 & 0.87431 & 1.90821 \\
\hline
\end{tabular}


Citation: Saeed E. Hemeda and Ali M. Abdallah, 2018. Non-Bayesian and Bayesian Estimation of Parameters for Burr Type III Distribution in Presence of K Outliers. Australian Journal of Basic and Applied Sciences., 12(4): 5-13.

Table 6: Bayesian parameters estimation for Burr type III in presence of two outliers with initial values; $k=2, \alpha=1, \lambda=2.8, \theta=2.5, \beta=2.9$.

\begin{tabular}{|c|c|c|c|c|c|c|}
\hline & Parameter & Mean & Bias & MSE & Skewness & Kurtosis \\
\hline \multirow[t]{2}{*}{$\overline{10}$} & $\alpha$ & 1.44393 & -0.44392 & 0.33083 & 1.16118 & 5.2397 \\
\hline & $\theta$ & 2.44284 & 0.067162 & 7.0861 & 6.9286 & 4.0506 \\
\hline \multirow[t]{2}{*}{20} & $\alpha$ & 1.21605 & -0.21605 & 0.06087 & -0.00002 & 0.99999 \\
\hline & $\theta$ & 4.3989 & -1.89890 & 4.63942 & 0.00006 & 0.99999 \\
\hline \multirow[t]{2}{*}{30} & $\alpha$ & 1.08545 & -0.08544 & 0.05728 & 0.250968 & 1.71735 \\
\hline & $\theta$ & 6.56182 & -4.06182 & 13.444 & 2.37688 & 7.10043 \\
\hline \multirow[t]{2}{*}{$\overline{40}$} & $\alpha$ & 1.70034 & 0.01367 & 0.09238 & -0.01286 & 1.99396 \\
\hline & $\theta$ & 2.70931 & -1.79842 & 0.54912 & 1.79852 & 1.88175 \\
\hline \multirow[t]{2}{*}{50} & $\alpha$ & 0.98076 & 0.01128 & 0.09628 & -0.00879 & 0.48999 \\
\hline & $\theta$ & 3.00566 & -1.8979 & 1.09871 & 2.99845 & 2.37233 \\
\hline \multirow[t]{2}{*}{60} & $\alpha$ & 0.99621 & 0.00896 & 0.00623 & 0.00175 & 1.10946 \\
\hline & $\theta$ & 3.00343 & -1.07821 & 1.00956 & 2.12924 & 4.65916 \\
\hline \multirow[t]{2}{*}{70} & $\alpha$ & 1.18071 & 0.00864 & 0.02019 & -0.12091 & 1.01464 \\
\hline & $\theta$ & 2.98765 & -0.39768 & 1.00083 & 2.00066 & 2.29766 \\
\hline \multirow[t]{2}{*}{80} & $\alpha$ & 1.09002 & 0.00199 & 0.00107 & 0.00763 & 1.12339 \\
\hline & $\theta$ & 2.70928 & -0.02129 & 0.02095 & 1.98277 & 2.47008 \\
\hline \multirow[t]{2}{*}{90} & $\alpha$ & 1.00560 & 0.00096 & 0.08192 & -0.00805 & 1.02261 \\
\hline & $\theta$ & 2.60081 & -0.01097 & 0.09087 & 1.00128 & 2.17554 \\
\hline \multirow[t]{2}{*}{100} & $\alpha$ & 1.20097 & 0.00815 & 0.00249 & 0.00011 & 1.15622 \\
\hline & $\theta$ & 2.09534 & -0.00794 & 0.00102 & 0.92946 & 1.32973 \\
\hline
\end{tabular}

Conclusion and Remarks:

This section provides conclusion and important remarks based on the results of the numerical studies for classical and Bayesian estimation methods.

1- In classical (non-Bayesian) study, the form of the model for the Burr type III distribution in presence of $k$ outliers is introduced and the classical estimators for the shape and the scale parameters of Burr type III distribution in the presence of one and two outliers are obtained using the moment and the MLE methods as indicated in the followings:

$\boldsymbol{a}$-The moment estimation numerical study is conducted for:

i. $\mathrm{k}=1, \alpha=0.4, \lambda=1.8, \theta=0.3, \beta=1.9$.

ii. $\mathrm{k}=2, \alpha=0.4, \lambda=1.8, \theta=0.3, \beta=1.9$

Results of bias, MSE, skewness and kurtosis of the parameter estimators of case (i) and case (ii) are introduced in Table (1) and Table (2) respectively. Results in table (1) and table (2) shows a negative relationship between the MSE of the estimators and the sample size; the MSE of the estimators decrease when the sample size $n$ increases. The MSEs or the variances in case (i) are smaller than in case (ii), so that the number of outliers has an effect on the parameter estimators. The biases of estimators increase when the number of outliers $k$ for a big sample sizen increses.

$\boldsymbol{b}$-The MLE numerical study is conducted for:

i. $k=1, \alpha=0.5, \lambda=2.8, \theta=0.4, \beta=2.9$.

ii. $\mathrm{k}=2, \alpha=0.5, \lambda=2.8, \theta=0.4, \beta=2.9$.

Table (3) shows results for bias, MSE, skewness and kurtosis of the parameter estimators for case (i), while table (4) shows results for case (ii).

For $\mathrm{k}=1,2$ the MSE of MLEs are less than the MSEs of the moment estimators for most values of $n$. This indicates the higher efficiency of MLEs compared to the moment estimators. Therefore, we suggest using the MLE method for classical parameters estimation of the Burr type III distribution in the case of one and two outliers.

2- In the Bayesian numerical study, it is noted that the posterior mean decreases when the sample sizen is increases for most values. The MSE of parameters decreases when the sample size nincreases. Similarly, the posterior variance of the parameters has the same behaviors when the sample size becomes large. Table (5) shows the results of bias, MSE, skewness and kurtosis of the parameter estimators in case $(k=1)$ and table $(6)$ shows the results in case $(k=2)$. The MSEs of most estimators in case $(k=1)$ are less than the MSEs in case $(k=2)$. The conclusion is that the number of outliers effects the parameter estimators such that; the biases of estimators are increasing with the increasing $k$ for big sample sizen.

Based on anaylsis of the results in Tables 1, 2, 3, 4, 5 and 6, compare to the classical estimation methods (moment and ML) we suggest using the Bayesian estimation method in estimating the parameters of the Burr type III distribution in presence of one and two outliers.

\section{REFERENCES}

Abd-Elfattah, A.M. and A.H. Alharbey, 2012. Bayesian estimation for Burr distribution type III based on trimmed samples. ISRN Applied Mathematics, 3: $1-18$.

Burr, I.W., 1942. Cumulative frequency functions. The annals of Mathematical Statistics, 13: 215-232.

Deiri, E., 2011. Estimation of parameters of gamma distribution in the presence of two outliers. Australian Journal of Basic \&Applied Science, 5: 1496-1502.

Dixit, U.J., 1989. Estimation of parameters of the gamma distribution in the presence of outliers. Communications in statistics-Theory and Methods, 18: 3071-3085.

Dixit, U.J., K.L. Moore and Barnett, 1996. On the estimation of power of the scale parameter of the exponential distribution in the presence of outliers generated from Uniform distribution. Metron, 54: 201-211.

Dixit, U.J. and P.E. Nasiri, 2001. Estimation of parameters of the exponential distribution in the presence of outliers generated from Uniform distribution. Metron, 49: 187-198.

Dixit, U.J. and M.J. Nooghabi, 2011. Efficient estimation in the Pareto distribution with the presence of outliers. Statistical Methodology, 8: $340-355$.

Ghanizadeh, A., H. Pazira and R. Lotfi, 2011. Classical estimations of the exponentiated gamma distribution parameters with presence of k outliers. Australian Journal of Basic and Applied Sciences, 5: 571-579.

Grubbs, F.E., 1969. Procedures for detecting outlying observations in samples. Technometrics, 11: 1-21. 
Gupta, P.K. and A.K. Singh, 2017. Classical and Bayesian estimation of Weibull distribution in presence of outliers. Cogent Mathematics, 4: 1-9.

Hassan, A.S., E.A. Elsherpieny and R.M. Shalaby, 2013. On the estimation of $\mathrm{P}(\mathrm{Y}<\mathrm{X}<\mathrm{Z})$ for Weibull distribution in the presence of $\mathrm{k}$ outliers. International Journal of Engineering Research and Applications, 3: 1727-1733.

Hossain, A.M. and S.K. Nath, 1997.Estimation of parameters in the presence of outliers for Burr XII distribution. Communications in Statistics, 26: 637-652.

Khokan, M.R., W. Bari and J.A. Khan, 2013. Weighted maximum likelihood approach for robust estimation: Weibull model (in the presence of outliers). Dhaka University Journal of Science, 61: 153-156.

Makhdoom, I., 2011. Estimation of the parameters of minimax distribution in the presence of outliers, International Journal of Academic Research, 3: 5005007.

Nasiri, P. and H. Pazira, 2010. Bayesian approach on the generalized exponential distribution in the presence of outliers. Journal of Statistical Theory and Practice, 4: 453-475.

Nooghabi, M.J., H.J. Nooghabi and P.F. Nasiri, 2009. Estimation of parameters of the gamma distribution in the presence of outliers generalized from uniform distribution. Pakistan Journal of Statistics, 25: 1. 